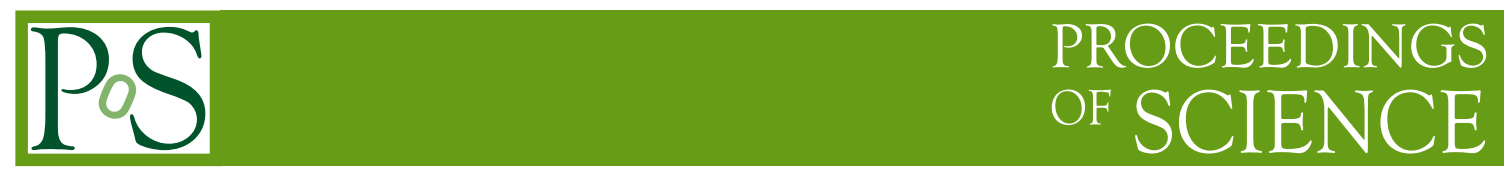

\title{
Spatial Resolution of the Belle II Silicon Vertex
} Detector

\author{
S. Halder ${ }^{j *}$, H. Aihara ${ }^{t}$, T. Aziz ${ }^{j}$, S. Bacher ${ }^{x}$, S. Bahinipati ${ }^{e}$, E. Barberio ${ }^{a}$, \\ Ti. Baroncelli ${ }^{a}$, To. Baroncelli ${ }^{a}$, A. K. Basith ${ }^{f}$, G. Batignani ${ }^{k}, l$, A. Bauer ${ }^{b}$,

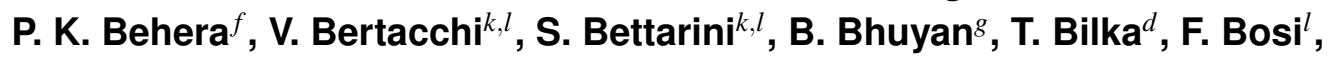 \\ L. Bosisio ${ }^{m, n}$, A. Bozek ${ }^{x}$, F. Buchsteiner ${ }^{b}$, G. Caria ${ }^{a}$, G. Casarosa ${ }^{k}, l$, M. Ceccanti $^{l}$, \\ D. Červenkov ${ }^{d}$, T. Czank ${ }^{s}$, N. Dash ${ }^{e}$, M. De Nuccio ${ }^{k, l}$, Z. Doležal ${ }^{d}$, F. Forti ${ }^{k, l}$, \\ M. Friedl ${ }^{b}$, R. Frühwirth ${ }^{b}$, B. Gobbo ${ }^{n}$, J. A. M. Grimaldo ${ }^{t}$, K. Hara ${ }^{u}$, T. Higuchi ${ }^{p}$, \\ C. Irmler ${ }^{b}$, A. Ishikawa ${ }^{s}$, H. B. Jeon ${ }^{v}$, C. Joo ${ }^{p}$, M. Kaleta $^{x}$, J. Kandra ${ }^{d}$, K. H. Kang ${ }^{v}$, \\ P. Kodys ${ }^{d}$, T. Kohriki ${ }^{u}$, I. Komarov ${ }^{n}$, M. Kumar ${ }^{h}$, R. Kumar ${ }^{i}$, P. Kvasnička ${ }^{d}$, C. La \\ Licata $^{m, n}$, K. Lalwani ${ }^{h}$, L. Lanceri ${ }^{m, n}$, J. Y. Lee ${ }^{w}$, S. C. Lee ${ }^{v}, \mathbf{Y} \mathbf{L i}^{c}$, J. Libby ${ }^{f}$, \\ T. Lueck ${ }^{k, l}$, P. Mammini ${ }^{l}$, A. Martini ${ }^{k l}$, S. N. Mayekar ${ }^{j}$, G. B. Mohanty ${ }^{j}$, T. Morii ${ }^{p}$, \\ K. R. Nakamura ${ }^{u}$, Z. Natkaniec ${ }^{x}$, Y. Onuki ${ }^{t}$, W. Ostrowicz ${ }^{x}$, A. Paladino ${ }^{p}$, E. Paoloni ${ }^{k} l$, \\ H. Park ${ }^{v}$, K. Prasanth ${ }^{j}$, A. Profeti ${ }^{l}$, K. K. Rao ${ }^{j}$, I. Rashevskaya ${ }^{n, o}$, P. K. Resmi ${ }^{f}$, \\ G. Rizzo $^{k, l}$, M. Rozanska ${ }^{x}$, D. Sahoo ${ }^{j}$, J. Sasaki ${ }^{t}$, N. Sato ${ }^{u}$, S. Schultschik ${ }^{b}$, \\ C. Schwanda ${ }^{b}$, J. Stypula ${ }^{x}$, J. Suzuki ${ }^{u}$, S. Tanaka ${ }^{u}$, H. Tanigawa ${ }^{t}$, G. N. Taylor ${ }^{a}$, \\ R. Thalmeier ${ }^{b}$, T. Tsuboyama ${ }^{u}$, P. Urquijo ${ }^{a}$, L. Vitale $^{m, n}$, K. Wan ${ }^{t}$, M. Watanabe ${ }^{q, r}$, \\ S. Watanuki ${ }^{s}$, I. J. Watson ${ }^{t}$, J. Webb ${ }^{a}$, J. Wiechczynski ${ }^{x}$, S. Williams ${ }^{a}$, H. Yin ${ }^{b}$ and \\ L. Zani ${ }^{k, l}$
}




\section{(Belle II SVD Group)}

${ }^{a}$ School of Physics, University of Melbourne, Melbourne, Victoria 3010, Australia

${ }^{b}$ Institute of High Energy Physics, Austrian Academy of Sciences, 1050 Vienna, Austria

${ }^{c}$ Peking University, Department of Technical Physics, Beijing 100871, China

${ }^{d}$ Faculty of Mathematics and Physics, Charles University, 12116 Prague, Czech Republic

${ }^{e}$ Indian Institute of Technology Bhubaneswar, Satya Nagar, India

${ }^{f}$ Indian Institute of Technology Madras, Chennai 600036, India

${ }^{g}$ Indian Institute of Technology Guwahati, Assam 781039, India

${ }^{h}$ Malviya National Institute of Technology, Jaipur 302017, India

${ }^{i}$ Punjab Agricultural University, Ludhiana 141004, India

${ }^{j}$ Tata Institute of Fundamental Research, Mumbai 400005, India

${ }^{k}$ Dipartimento di Fisica, Università di Pisa, I-56127 Pisa, Italy

${ }^{l}$ INFN Sezione di Pisa, I-56127 Pisa, Italy

${ }^{m}$ Dipartimento di Fisica, Università di Trieste, I-34127 Trieste, Italy

${ }^{n}$ INFN Sezione di Trieste, I-34127 Trieste, Italy

${ }^{o}$ Presently at TIFPA-INFN, Dipartimento di Fisica, Universit di Trento, I-38123 Trento, Italy

${ }^{p}$ Kavli Institute for the Physics and Mathematics of the Universe (WPI), University of Tokyo,

Kashiwa 277-8583, Japan

${ }^{q}$ Department of Physics, Niigata University, Niigata 950-2181, Japan

${ }^{r}$ Presently at Nippon Dental University, Niigata 951-8580, Japan

${ }^{s}$ Department of Physics, Tohoku University, Sendai 980-8578, Japan

${ }^{t}$ Department of Physics, University of Tokyo, Tokyo 113-0033, Japan

${ }^{u}$ High Energy Accelerator Research Organization (KEK), Tsukuba 305-0801, Japan

${ }^{v}$ Department of Physics, Kyungpook National University, Daegu 702-701, Korea

${ }^{w}$ Department of Physics and Astronomy, Seoul National University, Seoul 151-742, Korea

${ }^{x}$ H. Niewodniczanski Institute of Nuclear Physics, Krakow 31-342, Poland

E-mail: soumen.halderetifr.res.in

The Belle II experiment at the SuperKEKB collider in Japan will search for new sources of CP violation and indirectly probe new physics by studying the suppressed decays of beauty mesons, charm mesons and tau leptons. In these pursuits, the spatial resolution of the Belle II Silicon Vertex Detector (SVD) will play a key role. We report herein the spatial resolution of the SVD using simulated data for a simplified version of the Belle II detector.

The 27th International Workshop on Vertex Detectors - VERTEX2018

22-26 October 2018

MGM Beach Resorts, Muttukadu, Chennai, India

\footnotetext{
*Speaker.
} 


\section{Introduction}

Belle II at SuperKEKB in Tsukuba, Japan will search for new sources of CP violation and signatures of new physics by studying the suppressed decays of beauty mesons, charm mesons and tau leptons. The experiment is located at an interaction point of the SuperKEKB $e^{+} e^{-}$collider, which is operating at a center-of-mass energy near the $\Upsilon(4 S)$ resonance. The positron and electron beams have unequal energies of 4 and $7 \mathrm{GeV}$, respectively, which make the $\Upsilon(4 S)$ boosted in the lab frame with a Lorentz factor $\beta \gamma=0.28$ that is about two-thirds of that in Belle. The SuperKEKB, aims to deliver collision data at an unprecedented luminosity of $8 \times 10^{35} \mathrm{~cm}^{-2} \mathrm{~s}^{-1}, 40$ times higher than its predecessor (KEKB), by a sizable decrease in the beam cross-section and a moderate increase in the beam current.

At Belle II, the precise determination of decay vertex and low-momentum tracking are crucial to indirectly probe new physics. The reconstruction of charged particles near the interaction point is accomplished by a six-layer VerteX Detector (VXD), which consists of two layers, layer 1 and 2, of PiXel Detector (PXD) at radii 1.4 and $2.2 \mathrm{~cm}$, and four layers, layer 3 to 6, of Silicon Vertex Detector (SVD) at radii 3.9, 8.0, 10.4 and $13.5 \mathrm{~cm}$. The PXD and SVD are comprised of depleted p-channel field effect transistor pixel sensors and Double-sided Silicon Strip Detectors (DSSDs), respectively. The lower $\beta \gamma$ factor causes a smaller separation between the two B mesons compared to Belle; however thanks to a thinner beam pipe, the VXD is nearer to the interaction point and as a consequence, the SVD along with the PXD gives a better vertex measurement than Belle.

\section{The Silicon Vertex Detector}

The Belle II SVD consists of a number of DSSD sensors that are cylindrically arranged around the interaction point. Each DSSD sensor is either rectangular or trapezoidal having $300-320 \mu \mathrm{m}$ thickness with n-type bulk whose one side is doped by acceptor (called strips) and the other side is doped by donor, with strips being perpendicular to the former. The acceptor implanted side, called $\mathrm{p}$ or $\mathrm{U}$ side, having longer strips parallel to the length of the sensor, measures the $r-\phi$ coordinate. The donor implanted ( $\mathrm{n}$ or $\mathrm{V}$ side) shorter strips measure the $z$ coordinate. Layer-3 sensors have readout pitch $50 \mu \mathrm{m}$ in the $r-\phi$ plane and $160 \mu \mathrm{m}$ along the $z$ axis. On the other hand, layer-4, 5 and 6 sensors have readout pitch $75 \mu \mathrm{m}$ in the $r-\phi$ plane and $240 \mu \mathrm{m}$ along the $z$ axis. To improve the spatial resolution, one floating strip is implemented between two readout strips. A detailed description of the SVD can be found in Ref. [1].

\section{Phase-2 Monte Carlo Samples}

For the measurement of spatial resolution we use $e^{+} e^{-} \rightarrow \mu^{+} \mu^{-}$events. About $500 \mathrm{pb}^{-1}$ of $e^{+} e^{-}$collision data, corresponding to 0.6 million dimuon events, were collected between FebruaryJune 2018 during the Phase-2 running of Belle II, which included a partial VXD with rest of the subdetectors fully installed. We report here the measurement of spatial resolution on a simulated 
data sample of 5.0 million $e^{+} e^{-} \rightarrow \mu^{+} \mu^{-}$events. This study on simulated events has been performed to optimise and validate the procedure that will be later repeated on data.

A track is reconstructed with hits from the tracking subdetectors, namely PXD, SVD and Central Drift Chamber (CDC). To remove potential bias, we exclude hits of the SVD layer for which we wish to determine the spatial resolution. The projection of SVD hits on either side is called SVD cluster. An SVD cluster is reconstructed using serial strips whose signal-to-noise ratio is greater than 5 . The cluster position is given by the charge-weighted strip position. Two additional requirements are imposed for selecting the track: the hits associated with the track must contain at least one layer PXD hit and one layer SVD hit.

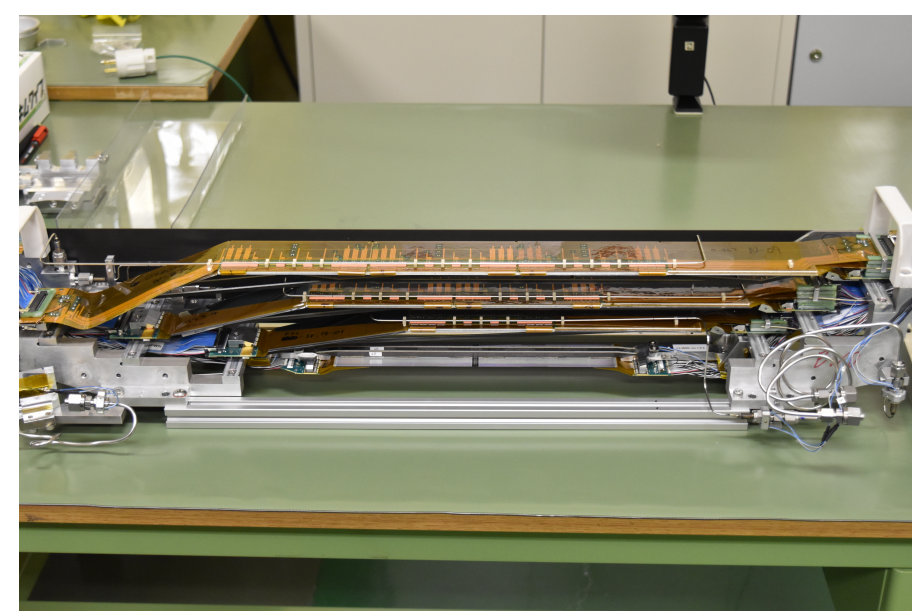

Figure 1: Phase-2 VXD subdetector, with one ladder per layer, is resting on a table before installation. The innermost layers, PXD layers 1 and 2, and SVD layer 3 are barely visible.

\section{Measurement of Spatial Resolution}

The spatial resolution of each sensor is extracted from the residual distribution, where the residual is the difference between the measured hit position and the position of track extrapolated to the sensor plane or the intercept position. The residual distribution has contributions from the uncertainty associated to the hit position (the spatial resolution ), $\sigma_{\text {hit }}$ and the uncertainty of the intercept position, $\sigma_{\text {track }}$. We can safely assume, at first order, that the hit position and intercept position are not correlated, and $\sigma_{\text {hit }}$ is calculated as the square root of the quadrature difference between $\sigma_{\text {res }}$ and $\sigma_{\text {track }}$ i.e.,

$$
\sigma_{\text {hit }}=\sqrt{\left(\sigma_{\text {res }}\right)^{2}-\left(\sigma_{\text {track }}\right)^{2}}
$$

where $\sigma_{\text {res }}$ is the residual distribution width. The residual width is extracted by fitting the residual distribution with a sum of two Gaussian functions of common mean and then calculating the weighted average of the Gaussian widths with the relative fraction of events fitted with each Gaus- 
sian. The fit function is given by

$$
f(x)=\frac{s}{\sqrt{2 \pi} \sigma_{1}} e^{-\frac{(x-\mu)^{2}}{2 \sigma_{1}^{2}}}+\frac{1-s}{\sqrt{2 \pi} \sigma_{2}} e^{-\frac{(x-\mu)^{2}}{2 \sigma_{2}^{2}}},
$$

where $\mu$ is the common mean, $\sigma_{1}$ and $\sigma_{2}$ are the widths of two Gaussians, and $s$ is the fraction of the first Gaussian. Thus, the effective width is $\sigma^{2}=s \sigma_{1}^{2}+(1-s) \sigma_{2}^{2}$. The error on track extrapolation or $\sigma_{\text {track }}$ is returned by the Kalman filter used to extrapolate the track to the sensor plane.

Figure 2 is an example of the residual distribution for the central sensor of the 6th layer, for clusters composed of a single strip.
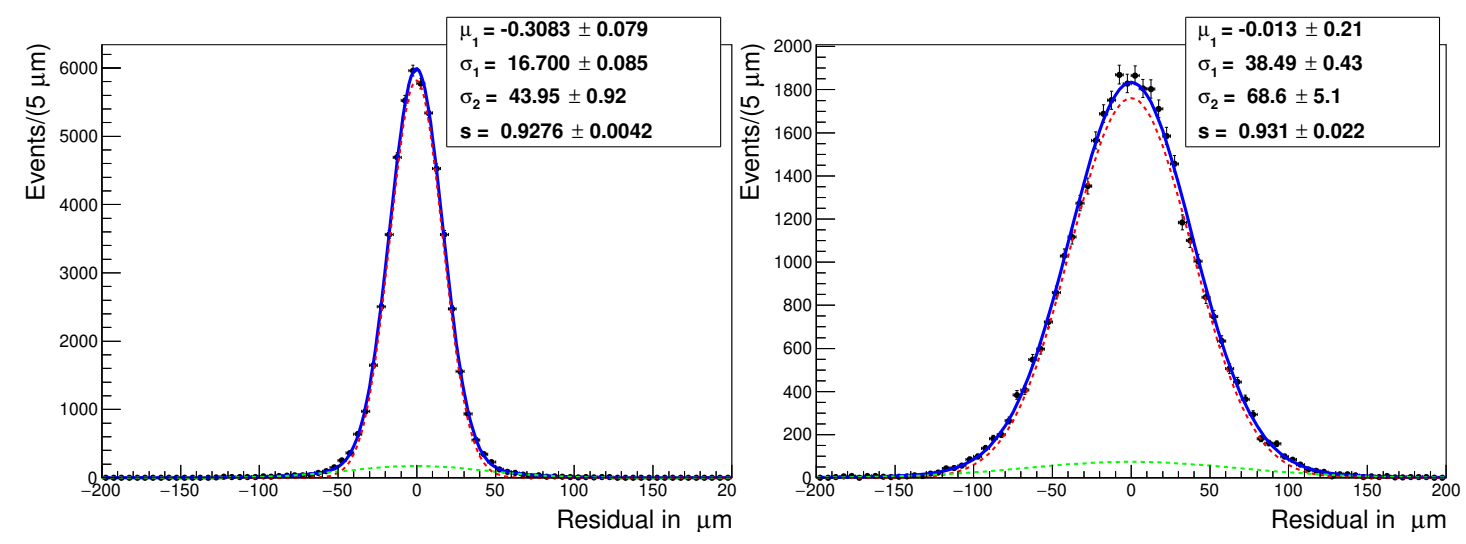

Figure 2: Residual distribution for the $6^{\text {th }}$ layer U-side (left) and V-side (right) with cluster size one.

Using the simulated sample, we find that the $\sigma_{\text {track }}$ value returned by the extrapolation is underestimated, therefore we calculate $\sigma_{\text {track }}$ using the true position of the intercept of the track with the sensor. Basically, we fit the distribution of true residual i.e., the difference in the intercept and true-hit position, to the sum of two Gaussian functions with common mean, and take its effective width as $\sigma_{\text {track }}$ (as described earlier). We will follow the same procedure for real data, except that as we do not have truth information there, the estimated truth information from the MC sample can be used. The statistical uncertainty associated to $\sigma_{\text {hit }}$ is

$$
\sqrt{\left[\frac{\sigma_{\text {res }}}{\sigma_{\text {hit }}} \delta\left(\sigma_{\text {res }}\right)\right]^{2}+\left[\frac{\sigma_{\text {track }}}{\sigma_{\text {hit }}} \delta\left(\sigma_{\text {track }}\right)\right]^{2}},
$$

with $\delta\left(\sigma_{\text {res }}\right)$ and $\delta\left(\sigma_{\text {track }}\right)$ being obtained from the fitting. 
Table 1: Spatial resolutions for different sensors of layer 3-6 for both $\mathrm{U}$ and $\mathrm{V}$ sides.

\begin{tabular}{|c|c|c|c|r|r|}
\hline Layer & Side & $\begin{array}{c}\text { Readout } \\
\text { pitch (p) } \\
\text { in } \mu m\end{array}$ & $\begin{array}{c}\text { Digital } \\
\text { resolution } \\
=\frac{\mathrm{p}}{2 \sqrt{12}} \\
\text { in } \mu m\end{array}$ & \multicolumn{2}{|c|}{$\begin{array}{c}\sigma_{\text {hit }} \\
\text { in } \mu m \\
\text { for cluster size }\end{array}$} \\
\hline 3 & $\mathrm{U}$ & 50 & 7.21 & one & two \\
\hline 3 & $\mathrm{~V}$ & 160 & 23.09 & $17.86 \pm 0.08$ & $14.66 \pm 0.19$ \\
4 & $\mathrm{U}$ & 75 & 10.82 & $10.0 \pm 0.09$ & $6.58 \pm 0.12$ \\
4 & $\mathrm{~V}$ & 240 & 34.64 & $26.83 \pm 0.17$ & $19.55 \pm 0.28$ \\
5 & $\mathrm{U}$ & 75 & 10.82 & $10.01 \pm 0.34$ & $6.53 \pm 0.22$ \\
5 & $\mathrm{~V}$ & 240 & 34.64 & $26.84 \pm 0.26$ & $18.77 \pm 0.43$ \\
6 & $\mathrm{U}$ & 75 & 10.82 & $11.56 \pm 0.51$ & $8.52 \pm 0.47$ \\
6 & $\mathrm{~V}$ & 240 & 34.64 & $27.16 \pm 1.77$ & $20.44 \pm 0.88$ \\
\hline
\end{tabular}
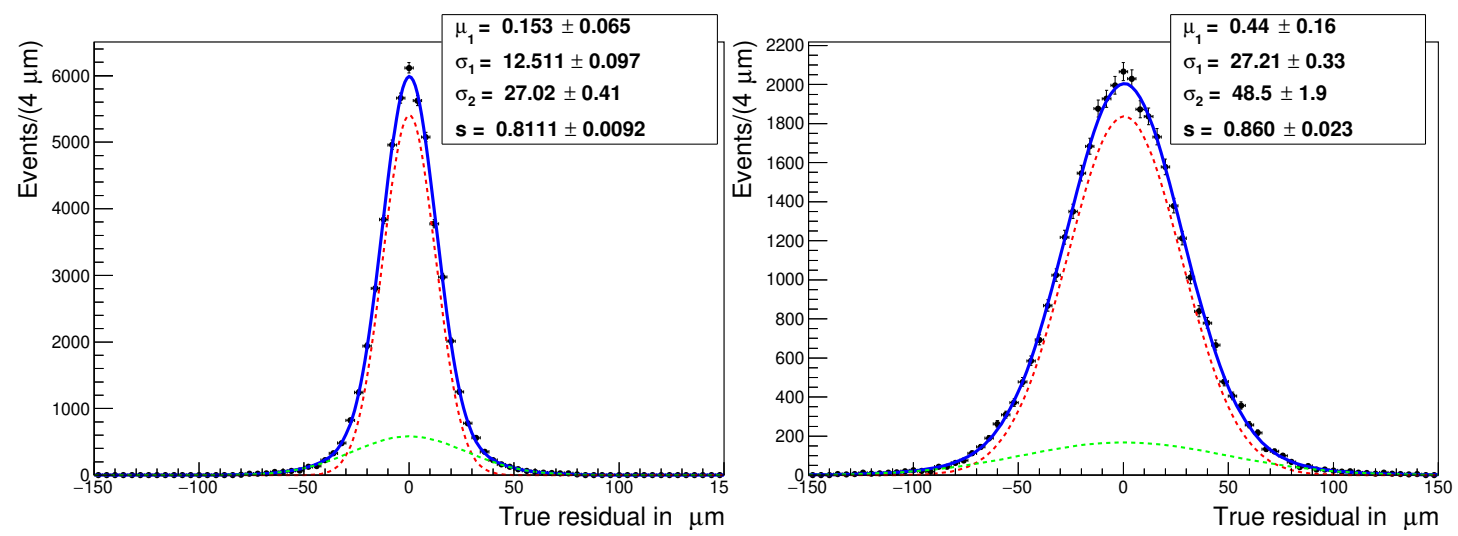

Figure 3: True residual distribution for the $6^{\text {th }}$ layer $\mathrm{U}$-side (left) and V-side (right) with cluster size one.

In figure 3 we show an example of the true residual distribution for the central sensor of the $6^{\text {th }}$ layer, for clusters composed of a single strip.

The estimated spatial resolution for each layer of the SVD is listed in Table 1. The factor of 2 in the digital resolution expression takes into account that there is one floating strip between two readout strips. The spatial resolution for multistrip cluster is better than single-strip cluster. This is because the position of multistrip cluster is estimated not only with the strip position but also using the information of charge collected by the strip. Thanks to this additional information multistrip cluster attains a better spatial resolution than single-strip one. Further, we find that the spatial resolution for single-strip cluster is better than the digital resolution. This is because in the former case the cluster position is calculated using the additional information that the strips beside the fired one have signal-to-noise ratio below threshold. Smaller cluster position residuals on the $\mathrm{U}$ side reflect the smaller strip pitch as well as a better estimation of track parameters compared to the $\mathrm{V}$ side. 


\section{Summary}

We have presented a Monte Carlo based study to estimate the spatial resolution of the SVD. The spatial resolution of the multistrip cluster is better than the single-strip cluster, which is in turn better than the digital resolution. The overall resolution ranges from about $5 \mu \mathrm{m}$ in the $\mathrm{U}$ side to $20 \mu \mathrm{m}$ in the $\mathrm{V}$ side.

\section{Acknowledgments}

I thank the organizers of Vertex-2018 for giving me an opportunity to participate in this conference. This work is supported by MEXT, WPI, and JSPS (Japan); ARC (Australia); BMWFW (Austria); MSMT (Czechia); AIDA-2020 (Germany); DAE and DST (India); INFN (Italy); NRF2016K1A3A7A09005605 and RSRI (Korea); and MNiSW (Poland).

\section{References}

[1] T. Abe et al., Belle II Technical Design Report, arXiv: 1011.0352.

[2] T. Lueck et al., PoS VERTEX 2016 (2016) 056.

[3] R. Turchetta, Nucl. Instrum. Methods Phys. Res., Sect. A 